\title{
The Influence of Critical Success Factors on B2B Sales Performance and the Mediating Role of Social Intelligence
}

\author{
Samet AYDIN (Corresponding author) \\ Faculty of Business and Management Sciences, Maltepe University \\ Marmara Egitim Koyu, Maltepe, Istanbul, Turkey
}

Tel: 90-216-6261050. E-mail: sametaydin@hotmail.com

Erdogan Koc

Faculty of Economics and Administrative Sciences, Bandirma Onyedi Eylul University

Bandirma, Balikesir, Turkey

Tel: 90-266-7380945. E-mail: erdogankoc@yahoo.com

Nihat Kaya

Faculty of Business Administration, Gebze Technical University

Gebze, Kocaeli, Turkey

Tel: 90-262-6051000. E-mail: nkaya@gtu.edu.tr

Received: July 19, 2017 Accepted: July 29, 2017 Published: August 15, 2017

doi:10.5296/bms.v8i2.11563～URL: https://doi.org/10.5296/bms.v8i2.11563

\begin{abstract}
In this study; the characteristics of sales representatives related to their sales performance such as learning orientation, customer orientation, intrinsic motivation, hardworking and technical expertise were defined as "Critical Success Factors in Selling" and the mediating role of social intelligence between these and sales performance were investigated. Data
\end{abstract}




\section{Macrothink}

Business Management and Strategy ISSN 2157-6068 2017, Vol. 8, No. 2

collected from 376 sales representatives from 125 different companies showed that social intelligence has strong influence over sales performance and plays a mediating role between critical success factors in selling and sales performance.

Keywords: Social Intelligence, Sales Performance, Learning Orientation, Customer Orientation, Intrinsic Motivation 


\section{Introduction}

The relational perspective in sales and marketing started with the development of business-to-business marketing (Ribeiro et al., 2015). Salespeople are vital for forming business value as they are in a position to understand the customer's value drivers, communicate products' value propositions, and provide customer insights to their own business firms (Blocker et al., 2012; Hohenschwert \& Geiger, 2015). The conceptualization of value creation in business relations requires the establishment of interaction processes in which relationship value is created (Corsaro \& Snehota, 2010; Grönroos, 2010). With this in mind business-to-business (B2B) firms invest millions of dollars annually to establish and operate better sales force systems and technologies that enhance productivity, communications, and customer relationships (Rodriguez \& Honeycutt, 2011).

The traditional understanding of sales function meant that sales people primarily had to just stimulate demand by persuading customers to buy products and services of the firm. Hence, the primary focus of the sales function used to be conceived as to make sales and contribute to the firm's short-term results by using selling techniques, such as hard selling (Chu et al., 1995; Albers et al., 2015).

However, the modern and marketing oriented view of sales emphasizes the problem solving role of the sales activity. In this role the sales function relates both to the needs of the customer and the business firm, and matches available product and service offerings to the customer's needs (Yu et al., 2015). The problem-solving role of sales rests on the assumption that salespeople have the knowledge of appropriate solutions to customers' problems. Sales staff play a crucial role for value creation in B2B relationships through day-to-day interactions with customers (Hohenschwert \& Geiger, 2015). The relational perspective of value developed over the past years in B2B marketing literature emphasizes an even greater role for salespeople in terms of influencing buyers' perceptions of value through interactions (Haas et al., 2012).

Additionally, the investigation of the factors influencing sales performance is also important as running sales departments are getting increasingly highly costlier (Sujan et al., 1988; Sabnis et al., 2013; Zampetakis, 2014). In addition to the salaries of sales, a variety of costs such as travelling and accommodation necessitate the recruitment and maintenance of only high performing sales staff (Deeter-Schmelz \& Sojka, 2007).

For this reason management researchers have concentrated their efforts over the years on studying the factors which influence salesperson performance (Churchill et al., 1985; Evans et al., 2012; Terho et al., 2015). Various studies have been carried out to assess a number of variables that relate to sales representatives' physical characteristics such as height, attractiveness, weight and age; personality characteristics such as gregariousness, extroversion/introversion, self/other orientation; and other miscellaneous characteristics such as intelligence, similarity to buyers, level of education etc. (Rozell et al., 2006; Alhouti et al., 2014). In general, researchers tend to agree that role perception, aptitude, skill level and 
motivation level are some of the most important determinants of salesperson performance (Churchill et al., 1985).

Previous studies confirmed that sales representatives' learning orientation (Sujan et al., 1994; Silver et al., 2006), customer orientation (Franke \& Park, 2006; Schwepker \& Good, 2012), intrinsic motivation (Oliver \& Anderson, 1994; Bodla \& Naeem, 2014), hard-working (Brown \& Peterson, 1994; Silver et al., 2006), and technical expertise (Plank \& Reid, 2010; Verbeke et al., 2010) appear to have positive influence on sales performance. Hence these may be considered as input factors for sales performance and called "the critical success factors in selling”.

A review of sales performance literature also shows that the social intelligence construct has been somehow ignored. Instead, similar constructs like emotional intelligence (Deeter-Schmelz \& Sojka, 2003; Rozell et al., 2006), interpersonal skills (Rentz et al., 2002), and empathy (Spiro \& Weitz, 1990; Plank \& Reid, 2010; Agnihotri \& Krush, 2015) have been quite often investigated from the perspective of sales performance.

Based on the above this study aims to explore the influence of learning orientation, customer orientation, intrinsic motivation, hardworking and technical expertise as critical success factors in selling and the mediating role of social intelligence on sales performance. Below in the literature review section why each of the variables has been included are explained.

\section{Introduction}

\subsection{Social Intelligence}

As mentioned briefly above a review of the sales performance literature shows that social intelligence construct has been largely ignored. Studies examining sales professionals' behaviors in relation to managing customers suggest that relationship management can be a significant factor in determining sales performance (Anderson \& Oliver, 1987; Cravens et al., 1993; Hunter \& Perreault, 2007). As most markets are increasingly becoming more competitive and customers are becoming more sophisticated and demanding and less loyal, the role of salespeople has become more important in terms of developing good customer relationships. Salespeople play a key role not only in terms of customer relationship management but also in terms of understanding, creating, communicating and delivering value to customers, which in turn are expected to increase the overall sales performance of the firm (Paparoidamis \& Guenzi, 2009).

According to Silvera et al. (2001) who also developed The Tromso Social Intelligence Scale, there are three dimension of social intelligence. These are:

i. Social Processing: Understanding others' feelings and thoughts in human relations and reading both verbal and behavioral messages.

ii. Social Skills: The level of success of a person in human relations. In other words, one's ability to get along with others, join in different social activities and adapt to different 
conditions.

iii. Social Awareness: Behaving in accordance with conditions, time and place, and responding properly to others behaviors.

Social intelligence differs from emotional intelligence in so much as it focuses on the impact that an individual has on others. Hence, social intelligence is rather relevant for sales performance. If one has high social intelligence then it is implied that $\mathrm{s} /$ he has high emotional intelligence as well, but not necessarily vice versa. During a typical working day sales representatives contact customers, suppliers, and colleagues from various functions of business. Thus social intelligence is crucial for sales representatives in terms of expressing themselves, persuading others, and achieving the desired outcomes during the interactions they engage (Riggio \& Lee, 2007). Social intelligence is often confused with the concept of emotional intelligence. Goleman (2006) suggests that social intelligence differentiates from emotional intelligence as it involves creating successful interactions in addition to understanding others.

Similarly Wawra (2009) stated that emotional intelligence is a prerequisite for social intelligence. Deeter-Schmelz and Sojka (2003) argue that emotional intelligence plays a crucial role in the success of sales representatives. Similarly, Rozell et al. (2006) supported this view. It's believed that sales representatives who have high emotional intelligence are also good at establishing interpersonal communications with customers. The establishment of interpersonal skills and social relationships are believed to lead to positive sales outcomes (Chakrabarty et al., 2014b). Moreover, by increasing adaptive selling behavior emotional intelligence influences the sales performance positively. Giacobbe et al. (2006) found that empathy during sales interactions helped sales representatives to establish and show adaptive behaviors during sales interactions. Rapport building is also crucial for sales representatives (Spiro and Weitz, 1990). According to Chakrabarty et al. (2014a) together with understanding non-verbal cues, rapport building contributes significantly to sales performance. Rentz et al. (2002) suggested that interpersonal skills are highly needed for effectiveness in sales as these skills enable sales representatives to build successful relations. Similarly, Castleberry and Shepherd (1993) and Comer (1999) stated that active listening skills are also important for each individual sales task. Based on the above the following hypotheses have been developed:

$H_{1 a}$ : There is a positive relation between social processing dimension of sales representatives' social intelligence and their sales performance.

$H_{1 b}$ : There is a positive relation between social skills dimension of sales representatives' social intelligence and their sales performance.

$H_{1 c}$ : There is a positive relation between social awareness dimension of sales representatives' social intelligence and their sales performance.

\subsection{Learning Orientation}


Marketing scholars have suggested that learning is central to creation of sustainable competitive advantage and enhancing business performance in today's fast changing and competitive marketplace (Chonko et al., 2003; Koc, 2009; Bell et al., 2010; Park et al., 2007). As many positive outcomes of learning generally accrue in the medium to long run, as opposed to the short run, many businesses may approach training and learning activities rather cautiously (Zhou et al., 2015). In particular, sales managers tend to concentrate on short term objectives and try to get out as much as possible from their sales staff in the short-run (Sujan et al., 1994; Wang \& Netemeyer, 2002).

Learning orientation of sales staff can show how much effort they are willingly prepared to exert for developing themselves to gain new information and update their skills (Dweck \& Leggett, 1988; Vande Walle et al., 1999; Wang \& Netemeyer, 2002; Evans et al., 2012). Learning oriented sales representatives tend to have an intrinsic desire to become better in their roles and usually are eager for taking challenging tasks and choosing unusual methods that provide them opportunities for new experiences (Sujan et al., 1994). Learning oriented sales people also see failures as learning opportunities. They tend adapt to changes more quickly, be not afraid of failures and do not easily give up when they encounter failures (Dweck \& Leggett, 1988; Ahearne et al., 2010).

Sales staff with learning orientation are more likely to try and find more effective and more efficient ways of executing regular tasks. As a consequence, sales staff with learning orientation become more satisfied with their jobs in the long run (Sujan et al., 1994). In order to establish organization-wide learning businesses are required to create a climate for personal learning (Sinkula et al., 1997; Calantone et al., 2002; Boyer et al., 2014). Creating a climate of organizational and personal learning is expected to support sustainable competitive advantage (Kohli et al., 1998; McFarland, 2003; Wei et al., 2013).

In a number of studies a positive correlation between learning orientation and sales performance were found (Sujan et al., 1994; Silver et al., 2006). However, yet in some others no significant relationship was found (Kohli et al., 1998; VandeWalle et al., 1999). Wang and Netemeyer (2002) argued that if learning oriented sales representatives given autonomy they may produce excellent results. Based on the above, the following hypothesis has been formulated:

$\mathrm{H}_{2}$ : There is a positive relation between learning orientation of sales representatives and their sales performance.

\subsection{Customer Orientation}

Contrary to product orientation, customer orientation requires all the activities of a business to be geared towards customers' needs and expectations (Strong \& Harris, 2004). In a customer oriented firm employees are continuously expected to collect information about the target market and translate this information into customer value (Slater \& Narver, 1995; Koc, 2003). Consumer orientation enables the establishment of long term relationships with customers. Thus, customer oriented salespeople not only sell their products but also try to 
build strong and sustainable relationships with their target customers (Schwepker, 2003). Furthermore, customer-oriented sales staff participate the new product development process actively and find final solutions to customers’ problems (Schwepker \& Good, 2012).

According to Saxe and Weitz (1982) customer oriented sales representatives are expected to (a) facilitate customers' purchase decisions which will meet their needs, (b) help their customers determine and assess their needs properly, (c) present products or services according to these needs, explain the facts of the products transparently, (d) avoid giving wrong or missing information and (e) avoid high-pressure sales situations.

A sales representative with customer orientation can assess her/his proposal from customers' perspective. Customer oriented sales people are able to judge whether promises made can be met by the business firm (Pettijohn et al., 2007). Customer oriented sales representatives are expected to explain the limitations and disadvantages of their products clearly to their customers. In so doing they can gain their customers' trust (Schwepker, 2003; Bateman \& Valentine, 2015) and they can prevent dissatisfaction in the long term (Saxe \& Weitz, 1982). According to Veiera et al. (2014) sales representative's performance is strengthened by simultaneous interrelated improvements in customer trust, satisfaction, and commitment.

It could be stated that customer orientation pays in the long run than in the short run (Joshi \& Randall, 2001). For this reason businesses are advised to concentrate their efforts and finances on building customer orientation. Franke and Park's study (2006) found that customer orientation has a strong correlation with sales performance. Likewise, Wachner et al. (2009) and Schwepker and Good's (2012) studies also support this view. Based on the above the following hypothesis has been developed:

H3: There is a positive relation between customer orientation of sales representatives and their sales performance.

\subsection{Intrinsic Motivation}

Motivation can be defined as a psychological state that initiates or directs behaviors towards a specific target by influencing an individual's cognitive choices (Brown \& Peterson, 1994). Hence, motivation can be thought of as the level of employees' willingness to spend effort to achieve organizational targets (Latham \& Ernst, 2006; Hartmann \& Slapničar, 2012). Intrinsic motivation is the motivation that comes from employee's personal beliefs, targets and needs (Bassett-Jones \& Lloyd, 2005; Chakrabarti et al., 2014a).

As the motivation construct can be instrumental in explaining the reasons behind why sales people behave differently in particular situations, sales managers have always been interested in understanding the concept of motivation (Walker et al., 1977; Churchill et al., 1985; Anderson \& Oliver, 1987). Many studies show that motivated salesperson work harder and produce more outcomes (Sujan, 1986; Weitz et al., 1986; Miao \& Evans, 2014). Based on the above the following hypothesis has been formulated: 
$\mathrm{H}_{4}$ : There is a positive relation between intrinsic motivation of sales representatives and their sales performance.

\subsection{Hard-Working}

Being hard-working is one of the prerequisites of sales profession (Sujan, 1986; Brown \& Peterson, 1994; Vande Walle et al., 1999; Krishnan et al., 2002). Hard-working sales representatives are more likely put greater effort and spend more time in sales related tasks compared with others. When a hard-working sales representative encounters a problem, or a short term failure, s/he tends to work harder to achieve, rather than to give up (Sujan et al., 1994; Krishnan et al., 2002).

Hard-working sales representatives tend to use their time more efficiently and make more customer calls while others spend more with one customer and waste more of their time with personal matters. Studies in sales literature indicate the correlation of being hard-working sales representative and sales performance (Silver et al., 2006; Chakrabarti et al., 2014b). Sujan et al. (1994) suggest that hard-working sales representatives are more effective in repeat sale tasks. Based on the above following hypothesis has been developed:

$H_{5}$ : There is a positive relation between hard-working of sales representatives and their sales performance.

\subsection{Technical Expertise}

Technical expertise is about sales representatives' ability to use their knowledge and experience to reach ideal solutions (Sujan et al., 1988; Bradford et al., 2010; Ko \& Dennis, 2013). Each member of the sales team is expected to have a sufficient level of knowledge of the products or services they offer to customers. Basic knowledge in terms of technical expertise may consist of information regarding the specifications of products or services, technical details for the proposed solutions and the processes regarding pre-sales or post-sales activities. In addition to these, all sales staff are normally expected to have sufficient knowledge of the potential target markets they serve, current market and customer trends and direct and indirect competitors (Sujan et al., 1988). Technical expertise allows sales representatives to find better and quicker solutions to customers' problems (Pettijohn et al., 2007). Additionally, technical expertise allows sales representatives to categorize their customers and find solutions accordingly (Weitz et al., 1986).

Sales representatives with technical expertise are more able to close sales (Sangtani \& Murshed, 2013; Leigh et al., 2014). They also tend to have less stress in complex situations, operate better (Miao \& Evans, 2012), and become more successful (Boyer et al., 2012). Verbeke et al. (2010) and suggested that there was a positive relationship between technical expertise and sales performance. Based on the above following hypothesis has been developed:

$H_{6}$ : There is a positive relation between technical expertise of sales representatives and their sales performance. 


\section{Sales Performance}

While functions like production, accounting, $R \& D$, supply chain etc. generally add to the costs of the firm, marketing and sales activities generate revenues. Sales performance can be defined as the quantitative contributions of sales representatives towards business targets (Behrman \& Perreault, 1982; Piercy et al., 2007). Sales performance can also be defined in the form of increasing market share, reaching planned sales volumes, increasing sales revenues and selling high profit margin products (Babakus et al., 1996; Flaherty \& Pappas, 2004). Regarding the mediational relations of social intelligence, the hypothesis below are proposed.

H7: Dimensions of social intelligence (social processing: $H_{7 a}$, social skills: $H_{7 b}$, social awareness: $\mathrm{H}_{7 c}$ ) mediates the relation of learning orientation and sales performance.

H8: Dimensions of social intelligence (social processing: H8a, social skills: H8b, social awareness: $\left.H_{8 c}\right)$ mediates the relation of customer orientation and sales performance.

H9: Dimensions of social intelligence (social processing: $H_{9 a}$, social skills: $H_{9 b}$, social awareness: $H_{9 c}$ ) mediates the relation of intrinsic motivation and sales performance.

$H_{10}$ : Dimension of social intelligence (social processing: H10a, social skills: $H_{10 b}$, social awareness: $\mathrm{H}_{10 c}$ ) mediates the relation of hard-working and sales performance.

$H_{11}$ : Dimension of social intelligence (social processing: $H_{11 a}$, social skills: $H_{11 b}$, social awareness: $H_{11 c}$ ) mediates the relation of technical expertise and sales performance.

\section{Method}

The research was carried out with B2B sales representatives in Istanbul, Turkey. A survey has been prepared and sent out to B2B sales representatives determined on convenience sampling. A total of 376 surveys were collected from B2B sales representatives working in 125 different companies.

Of the participants $80.6 \%$ of them were male and $19.4 \%$ were female. In terms of the level of education of the participants $69.7 \%$ of the participants had bachelor's degrees, $15.7 \%$ have master's degrees and $14.6 \%$ graduates of high schools or technical schools. Of the sales representatives $39.6 \%$ were between the ages of 26 and 32, $35.9 \%$ were between 33 and 39, $18.9 \%$ were older than 40 and $3.7 \%$ were below the age of 25 . In terms of years' of experience $30.3 \%$ of the participants had 5 to 9 years', $23.1 \%$ had 10 to14 years', and $18.6 \%$ had 15 to19 years' experience. Of the participants $17.3 \%$ had less than 4 years' and 10.6 had more than 20 years' experience.

\subsection{Measuring Validation}

Constructs were measured with five-point Likert scales which were found to be valid and reliable in previous studies. For measuring social intelligence "The Tromso Social Intelligence Scale” by Silvera et al. (2001) was used. Sales performance was measured with 
the seven items taken from Sujan et al. (1994). Learning orientation scale was also adopted from Sujan et al. (1994). For measuring customer orientation five items from Saxe and Weitz (1982) were used. The intrinsic motivation of the sales people were measured with three items developed by Anderson and Oliver (1987). Hard-working scale consists three items which were taken from Sujan et al. (1994). Finally for technical expertise six items were taken from Rentz et al. (2002).

In order to check the validity and the reliability, confirmatory factor analyses were conducted. Partial least squares regression (PLS) method, which is reported to give adequate results even in small samples (Chin, 1998) was chosen. The SmartPLS 3 software was used (Ringle et al., 2015). Even though PLS method does not have a minimum limit for sampling it is advised to have at least ten times of the maximum items that belong to a construct (Chin, 1998; Hair et al., 2011). In the study, the largest scale was social intelligence with 21 items. As the sample size was larger than ten times of twenty one this condition was met $(\mathrm{N}=376>21 * 10)$. PLS algorithm was executed and factor loadings were checked. Items that had low factor loadings were omitted (Hair et al., 2011; Hair et al., 2014). Finally, as seen in Appendix A all factor loadings were over 0.60 . In order to test the statistical significance of confirmatory factor analysis bootstrap procedure was held with 1000 samples (Hair et al., 2014). Results indicated that all items were statistically significant $(t>2.57, \mathrm{p}<0.01)$. Convergent validity and discriminant validity were met by following the directives of Hair et al. (2014) according to Fornell and Larcker (1981) criterion. Composite reliability (CR) of all structures was over 0.70. The Cronbach's Alpha values were also over 0.70. These results can be seen in Table 1 .

Table 1. Factor correlations

\begin{tabular}{|c|c|c|c|c|c|c|c|c|c|c|c|c|c|c|c|c|}
\hline & Factor & $\begin{array}{l}\mathrm{Me} \\
\mathrm{an}\end{array}$ & SD & CR & $\begin{array}{l}\text { Cr. } \\
\alpha\end{array}$ & $\begin{array}{l}\text { AV } \\
\mathrm{E}\end{array}$ & $\begin{array}{l}\sqrt{ } \mathrm{A} \\
\mathrm{VE}\end{array}$ & 1 & 2 & 3 & 4 & 5 & 6 & 7 & 8 & 9 \\
\hline 1 & $\begin{array}{l}\text { Customer } \\
\text { Orientation }\end{array}$ & $\begin{array}{l}4.1 \\
00\end{array}$ & $\begin{array}{l}0.5 \\
57 \\
\end{array}$ & $\begin{array}{l}0.8 \\
36 \\
\end{array}$ & $\begin{array}{l}0.7 \\
40 \\
\end{array}$ & $\begin{array}{l}0.5 \\
62 \\
\end{array}$ & $\begin{array}{l}0.7 \\
50\end{array}$ & $\begin{array}{l}1.0 \\
00 \\
\end{array}$ & & & & & & & & \\
\hline 2 & $\begin{array}{l}\text { Technical } \\
\text { Expertise }\end{array}$ & $\begin{array}{l}3.7 \\
57\end{array}$ & $\begin{array}{l}0.6 \\
43\end{array}$ & $\begin{array}{l}0.8 \\
62\end{array}$ & & $\begin{array}{l}0.5 \\
57\end{array}$ & $\begin{array}{l}0.7 \\
46\end{array}$ & $\begin{array}{l}0.2 \\
93\end{array}$ & $\begin{array}{l}1.0 \\
00\end{array}$ & & & & & & & \\
\hline 3 & $\begin{array}{l}\text { Intrinsic } \\
\text { Motivation }\end{array}$ & $\begin{array}{l}4.2 \\
60\end{array}$ & $\begin{array}{l}0.6 \\
13\end{array}$ & $\begin{array}{l}0.8 \\
85\end{array}$ & & $\begin{array}{l}0.7 \\
21\end{array}$ & $\begin{array}{l}0.8 \\
49\end{array}$ & $\begin{array}{l}0.5 \\
42\end{array}$ & $\begin{array}{l}0.2 \\
49\end{array}$ & $\begin{array}{l}1.0 \\
00\end{array}$ & & & & & & \\
\hline 4 & $\begin{array}{l}\text { Learning } \\
\text { Orientation }\end{array}$ & $\begin{array}{l}4.2 \\
97\end{array}$ & $\begin{array}{l}0.5 \\
72\end{array}$ & $\begin{array}{l}0.8 \\
77\end{array}$ & $\begin{array}{l}0.8 \\
27\end{array}$ & $\begin{array}{l}0.5 \\
88\end{array}$ & $\begin{array}{l}0.7 \\
67\end{array}$ & $\begin{array}{l}0.4 \\
29\end{array}$ & $\begin{array}{l}0.2 \\
59\end{array}$ & $\begin{array}{l}0.5 \\
63\end{array}$ & $\begin{array}{l}1.0 \\
00\end{array}$ & & & & & \\
\hline 5 & \begin{tabular}{|l|} 
Sales \\
Performance
\end{tabular} & $\begin{array}{l}4.1 \\
72\end{array}$ & $\begin{array}{l}0.5 \\
98\end{array}$ & $\begin{array}{l}0.8 \\
94\end{array}$ & $\begin{array}{l}0.8 \\
62\end{array}$ & $\begin{array}{l}0.5 \\
49\end{array}$ & $\begin{array}{l}0.7 \\
41\end{array}$ & $\begin{array}{l}0.4 \\
75\end{array}$ & $\begin{array}{l}0.2 \\
41\end{array}$ & $\begin{array}{l}0.5 \\
90\end{array}$ & $\begin{array}{l}0.4 \\
79\end{array}$ & $\begin{array}{l}1.0 \\
00\end{array}$ & & & & \\
\hline 6 & \begin{tabular}{|l|} 
Social \\
Processing
\end{tabular} & $\begin{array}{l}3.9 \\
66\end{array}$ & $\begin{array}{l}0.5 \\
24\end{array}$ & $\begin{array}{l}0.9 \\
04\end{array}$ & $\begin{array}{l}0.8 \\
77\end{array}$ & $\begin{array}{l}0.5 \\
75\end{array}$ & $\begin{array}{l}0.7 \\
58\end{array}$ & $\begin{array}{l}0.4 \\
84\end{array}$ & $\begin{array}{l}0.3 \\
68\end{array}$ & $\begin{array}{l}0.5 \\
19\end{array}$ & $\begin{array}{l}0.4 \\
07\end{array}$ & $\begin{array}{l}0.5 \\
25\end{array}$ & $\begin{array}{l}1.0 \\
00\end{array}$ & & & \\
\hline 7 & Social Skills & $\begin{array}{l}4.1 \\
12 \\
\end{array}$ & $\begin{array}{l}0.6 \\
11 \\
\end{array}$ & $\begin{array}{l}0.9 \\
27\end{array}$ & $\begin{array}{l}0.9 \\
07\end{array}$ & $\begin{array}{l}0.6 \\
44\end{array}$ & $\begin{array}{l}0.8 \\
02\end{array}$ & $\begin{array}{l}0.5 \\
20\end{array}$ & $\begin{array}{l}0.3 \\
84\end{array}$ & $\begin{array}{l}0.5 \\
93\end{array}$ & $\begin{array}{l}0.5 \\
04\end{array}$ & $\begin{array}{l}0.5 \\
80\end{array}$ & $\begin{array}{l}0.6 \\
34 \\
\end{array}$ & $\begin{array}{l}1.0 \\
00\end{array}$ & & \\
\hline 8 & $\begin{array}{l}\text { Social } \\
\text { Awareness }\end{array}$ & $\begin{array}{l}4.2 \\
63\end{array}$ & $\begin{array}{l}0.5 \\
89\end{array}$ & $\begin{array}{l}0.9 \\
24\end{array}$ & $\begin{array}{l}0.9 \\
04\end{array}$ & $\begin{array}{l}0.6 \\
36\end{array}$ & $\begin{array}{l}0.7 \\
97\end{array}$ & $\begin{array}{l}0.5 \\
94\end{array}$ & $\begin{array}{l}0.3 \\
24\end{array}$ & $\begin{array}{l}0.6 \\
07\end{array}$ & $\begin{array}{l}0.3 \\
71\end{array}$ & $\begin{array}{l}0.6 \\
06\end{array}$ & $\begin{array}{l}0.5 \\
92\end{array}$ & $\begin{array}{l}0.5 \\
71\end{array}$ & $\begin{array}{l}1.0 \\
00\end{array}$ & \\
\hline 9 & Hard-working & $\begin{array}{l}4.1 \\
78\end{array}$ & $\begin{array}{l}0.6 \\
57\end{array}$ & $\begin{array}{l}0.8 \\
85\end{array}$ & $\begin{array}{l}0.8 \\
06\end{array}$ & $\begin{array}{l}0.7 \\
21\end{array}$ & $\begin{array}{l}0.8 \\
49\end{array}$ & $\begin{array}{l}0.5 \\
43\end{array}$ & $\begin{array}{l}0.2 \\
48\end{array}$ & $\begin{array}{l}0.5 \\
68\end{array}$ & $\begin{array}{l}0.5 \\
19\end{array}$ & $\begin{array}{l}0.5 \\
42\end{array}$ & \begin{tabular}{|l|}
0.4 \\
51
\end{tabular} & $\begin{array}{l}0.5 \\
25\end{array}$ & $\begin{array}{l}0.5 \\
75\end{array}$ & $\begin{array}{l}1.0 \\
00\end{array}$ \\
\hline
\end{tabular}




\section{Method}

Multiple regression analysis was carried out test the hypotheses of the study. First, a regression model was built by taking the direct influences of all constructs on sales performance. Next, mediational regression analysis was carried out to test the mediating effects of social intelligence.

Regression results, stated in Table 2 , showed that social processing $(\beta=0.113 ; p<0.05)$, social skills $(\beta=0.187 ; p<0.01)$, social awareness $(\beta=0.256 ; p<0.01)$, learning orientation $(\beta=0.132$; $\mathrm{p}<0.01)$, intrinsic motivation $(\beta=0.149 ; \mathrm{p}<0.01)$ and hard-working $(\beta=0.094 ; \mathrm{p}<0.10)$ had significant influence on sales performance. On the other hand customer orientation $(\beta=-0.014$, $p>0.05)$ and technical expertise $(\beta=-0.045 ; p>0.05)$ were found to have insignificant influence on sales performance $(\mathrm{F}=45.416, \mathrm{p}<0.001)$.

Table 2. Regression analysis

\begin{tabular}{|l|l|l|}
\hline \multicolumn{3}{|l|}{ Dependent Variable: Sales Performance } \\
\hline Independent Variable & $\beta$ & $\mathrm{p}$ \\
\hline Social Processing & 0.113 & 0.030 \\
\hline Social Skills & 0.187 & 0.001 \\
\hline Social Awareness & 0.256 & 0.000 \\
\hline Learning Orientation & 0.132 & 0.005 \\
\hline Customer Orientation & -0.014 & 0.784 \\
\hline Intrinsic Motivation & 0.149 & 0.006 \\
\hline Hard-working & 0.094 & 0.064 \\
\hline Technical Expertise & -0.045 & 0.270 \\
\hline $\mathrm{R}$ & 0.705 & \multirow{2}{*}{0.000} \\
\cline { 1 - 1 } $\mathrm{R}^{2}$ & 0.497 & \multirow{2}{|l|}{} \\
\hline $\mathrm{F}$ & 45.416 & \\
\hline
\end{tabular}

According to the analysis $\mathrm{H}_{1}, \mathrm{H}_{1}, \mathrm{H}_{1 \mathrm{c}}, \mathrm{H}_{2}, \mathrm{H}_{4}$ and $\mathrm{H}_{5}$ were accepted but $\mathrm{H}_{3}$ and $\mathrm{H}_{6}$ were rejected. Mediating variables enter between independent and dependent variables and either decrease or exterminate the effect of independent variable (Baron and Kenny, 1986). To test the mediating effects PROCESS macro, developed by Hayes (2012) was used. According to mediational regression analysis, dimensions of social intelligence were found to take mediating roles between the critical success factors in selling and sales performance. Results can be seen in Table 3 to Table 7 .

Table 3. Learning Orientation and Sales Performance: Mediating of Social Intelligence

\begin{tabular}{|c|c|c|c|c|c|c|c|c|}
\hline Model & Independent Variable & Dependent Variable & $\mathrm{R}^{2}$ & $\mathrm{~F}$ & & $\beta$ & & $\mathrm{p}$ \\
\hline & Learning Orientation & Sales Performance & \begin{tabular}{|l|l|}
0.466 & 0.217
\end{tabular} & 103.931 & 0.000 & 0.466 & 0.19 & 0.000 \\
\hline \multirow{4}{*}{$H_{7 a}$} & Learning Orier & Social Proc & \begin{tabular}{|l|l|}
0.357 & 0.127 \\
\end{tabular} & & 0.000 & 0.357 & 7.389 & 0.000 \\
\hline & Social Processing & \multirow{3}{*}{ Sales Performance } & 0.599 & \multirow{2}{*}{104.184} & & 0.402 & 9.052 & \multirow{2}{*}{\begin{tabular}{|l|}
0.000 \\
0.000
\end{tabular}} \\
\hline & Learning Orientation & & 0 & & & 0.323 & & \\
\hline & & & Indil & & & 0.143 & & \\
\hline \multirow{4}{*}{$H_{7 b}$} & Learr & Skills & \begin{tabular}{|l|l|}
0.489 & 0.239 \\
\end{tabular} & 11 & 0.000 & 0.489 & \multirow{3}{*}{\multicolumn{2}{|c|}{\begin{tabular}{|l|l|}
10.845 & 0.000 \\
9.770 & 0.000 \\
5.173 & 0.000 \\
\end{tabular}}} \\
\hline & Social Skills & \multirow{3}{*}{ Sales Performance } & \multirow{2}{*}{0.614} & & \multirow{3}{*}{0.000} & 0.458 & & \\
\hline & Learning Orientation & & & & & 0.242 & & \\
\hline & & & Indirect Ef & & & 0.224 & & \\
\hline
\end{tabular}




\begin{tabular}{|l|l|l|l|l|l|l|l|l|l|}
\hline \multirow{3}{*}{$H_{7 c}$} & Learning Orientation & Social Awareness & 0.358 & 0.128 & 54.870 & 0.000 & 0.358 & 7.407 & 0.000 \\
\cline { 2 - 8 } & Social Awareness & Sales Performance & 0.654 & 0.428 & 139.494 & 0.000 & 0.491 & 11.714 & 0.000 \\
\cline { 2 - 8 } & Learning Orientation & & & & 0.291 & 6.930 & 0.000 \\
\cline { 2 - 7 }
\end{tabular}

Table 4. Customer Orientation and Sales Performance: Mediating of Social Intelligence

\begin{tabular}{|c|c|c|c|c|c|c|c|c|c|}
\hline Mode & Independent Variable & Dependent Variable & $\mathrm{R}$ & $\mathrm{R}^{2}$ & $\mathrm{~F}$ & & $\beta$ & $\mathrm{t}$ & $\mathrm{p}$ \\
\hline & Customer Orientation & Sales Performance & 0.446 & 0.199 & 92.922 & 0.000 & 0.446 & 9.640 & 0.000 \\
\hline \multirow{4}{*}{$H_{8 a}$} & Customer Orientation & Social Processing & 0.464 & 0.215 & 102.696 & 0.000 & 0.464 & 10.134 & 0.000 \\
\hline & Social Processing & \multirow{2}{*}{ Sales Performance } & 0.567 & 0.322 & 88398 & 0,000 & 0.395 & 8.209 & 0.000 \\
\hline & Customer Orientation & & \multirow{2}{*}{\multicolumn{3}{|c|}{$\frac{1}{\text { Indirect Effect }}$}} & & 0.263 & 5.455 & 0.000 \\
\hline & & & & & & & 0.183 & \multirow[b]{2}{*}{1109} & \\
\hline \multirow{3}{*}{$H_{8 b}$} & Customer Orientation & Social Skills & 0.497 & 0.247 & 122.977 & 0.000 & 0.497 & & 0.000 \\
\hline & Social Skills & \multirow{2}{*}{ Sales Performance } & \multirow{2}{*}{0.605} & \multirow{2}{*}{0.366} & \multirow{2}{*}{107.649} & 00000 & 0.471 & \multirow{2}{*}{$\begin{array}{l}9.911 \\
4.456 \\
\end{array}$} & \multirow{2}{*}{\begin{tabular}{|l|}
0.000 \\
0.000 \\
\end{tabular}} \\
\hline & Customer Orientation & & & & & & $\frac{0.212}{0.234}$ & & \\
\hline \multirow{4}{*}{$H_{8 c}$} & Customer Orientation & Social A & 0.580 & 0.336 & 189.19 & 0.000 & 0.580 & 13.75 & 0.000 \\
\hline & Social Awareness & \multirow{3}{*}{ Sales Performance } & \multirow{2}{*}{0.608} & \multirow{2}{*}{0.370} & \multirow{3}{*}{109.370} & م) & \multirow{3}{*}{\begin{tabular}{|l|}
0.507 \\
0.152 \\
\end{tabular}} & \multirow{3}{*}{\begin{tabular}{|l|}
10.049 \\
3.019 \\
\end{tabular}} & \multirow{3}{*}{\begin{tabular}{|l|}
0.000 \\
0.003 \\
\end{tabular}} \\
\hline & Customer Orientation & & & & & 000 & & & \\
\hline & & & \multicolumn{3}{|c|}{ Indirect Effect } & & & & \\
\hline
\end{tabular}

Table 5. Intrinsic Motivation and Sales Performance: Mediating of Social Intelligence

\begin{tabular}{|c|c|c|c|c|c|c|c|}
\hline Model & Independent Variable & Dependent Variable & $\mathrm{R}^{2}$ & $F$ & $\beta$ & & $\mathrm{p}$ \\
\hline & Intrinsic Motivation & Sales Performance & \begin{tabular}{|l|l|l|}
0.574 & 0.330 \\
\end{tabular} & $184.070 \mid 0.000$ & 0.574 & 13.567 & 0.000 \\
\hline \multirow{4}{*}{$H_{9 a}$} & Intrinsic Motivation & Social Processing & \begin{tabular}{|l|l|}
0.506 & 0.256 \\
\end{tabular} & \begin{tabular}{|l|l|}
128.628 & 0.000 \\
\end{tabular} & 0.506 & 11.341 & 0.000 \\
\hline & Social Processing & \multirow{3}{*}{ Sales Performance } & \multirow{2}{*}{0.399} & & 0.305 & 6.544 & 0.000 \\
\hline & Intrinsic Motivation & & & & 0.420 & 9.030 & 0.000 \\
\hline & & & Indirect Effe & & 0.154 & & \\
\hline \multirow{4}{*}{$H_{9 b}$} & Intrinsic Motivation & Social Skills & \begin{tabular}{|l|l|}
0.585 & 0.342 \\
\end{tabular} & \begin{tabular}{|l|l|}
194.237 & 0.000 \\
\end{tabular} & 0.585 & 13.937 & 0.000 \\
\hline & Social Skills & \multirow{3}{*}{ Sales Performance } & \multirow{2}{*}{0.418} & & 0.366 & 7.507 & 0.000 \\
\hline & Intrinsic Motivation & & & & 0.361 & 7.404 & 0.000 \\
\hline & & & Indirect Effe & & 0.213 & & \\
\hline \multirow{4}{*}{$H_{9 c}$} & Intrinsic Motivation & Social Awareness & \begin{tabular}{|l|l|}
0.601 & 0.361 \\
\end{tabular} & \begin{tabular}{|l|l|}
211.411 & 0.000 \\
\end{tabular} & 0.601 & 14.540 & 0.000 \\
\hline & Social Awareness & \multirow{3}{*}{ Sales Performance } & \multirow{2}{*}{0.654} & \multirow{3}{*}{139.385} & \multirow{3}{*}{\begin{tabular}{|l|}
0.391 \\
0.339 \\
0.235 \\
\end{tabular}} & 7.987 & \multirow{3}{*}{\begin{tabular}{|l|}
0.000 \\
0.000 \\
\end{tabular}} \\
\hline & Intrinsic Motivation & & & & & 6.919 & \\
\hline & & & Indirect Ef & & & & \\
\hline
\end{tabular}

Table 6. Hard-working and Sales Performance: Mediating of Social Intelligence

\begin{tabular}{|c|c|c|c|c|c|c|c|c|c|}
\hline Todel & Independent Va & Dependent Variable & $\mathrm{R}$ & $\mathrm{R}^{2}$ & $F$ & & $\beta$ & & p \\
\hline & Hard-working & Sales Performance & 0.509 & 0.259 & 130.826 & 0.000 & 0.509 & 11.438 & 0.000 \\
\hline \multirow{4}{*}{ Н10a } & Hard-working & Social Pro & 0.405 & & & 0.000 & 0.405 & 8.575 & 0.000 \\
\hline & Social Processing & \multirow{3}{*}{ Sales Performance } & \multirow{2}{*}{0.612} & \multirow{2}{*}{0.375} & \multirow{2}{*}{111.774} & & 0.372 & 8.304 & 0.000 \\
\hline & Hard-w & & & & & 0.000 & 0.358 & 8.001 & 0.000 \\
\hline & & & Indire & ect Eff & & & 0.151 & & \\
\hline \multirow{4}{*}{$H_{10 b}$} & Hard & So & 0.498 & 0.248 & & 0.000 & 0.498 & 11 & 0.000 \\
\hline & Social Skills & \multirow{3}{*}{ Sales Performance } & \multirow{2}{*}{0.631} & \multirow{2}{*}{0.398} & \multirow{2}{*}{123.170} & & 0.429 & 9.365 & \multirow{3}{*}{\begin{tabular}{|l|}
0.000 \\
0.000 \\
\end{tabular}} \\
\hline & Hard-working & & & & & & 0.295 & 6.371 & \\
\hline & & & Indi & & & & 0.214 & & \\
\hline H10c & Hard-n & $\mathrm{OC}$ & 0.56 & 1031 & & 80 & 0.564 & 13.2 & $\overrightarrow{01}$ \\
\hline
\end{tabular}




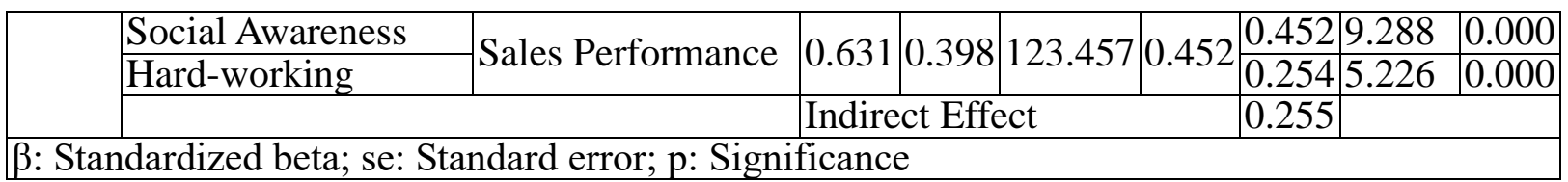

Table 7. Technical Expertise and Sales Performance: Mediating of Social Intelligence

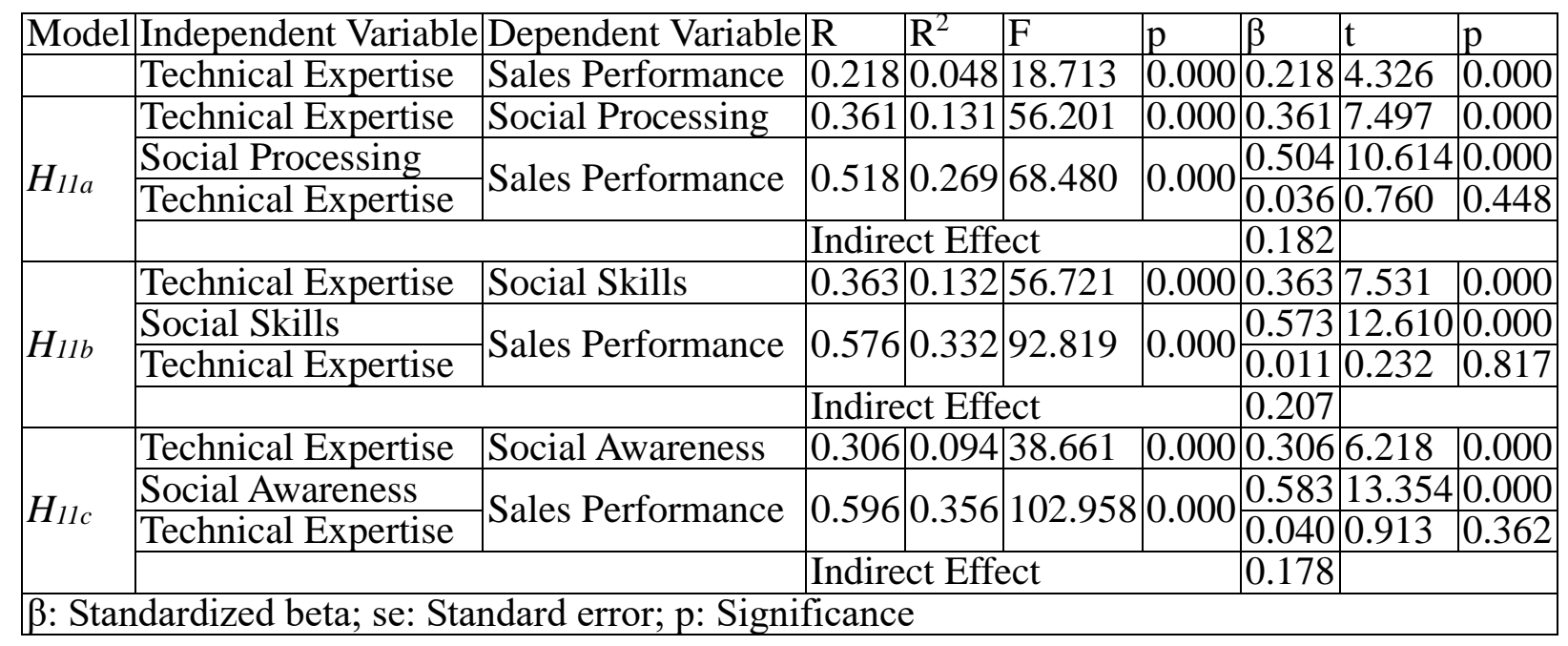

Analyses revealed that dimensions of social intelligence play mediating role between critical success factors in selling and sales performance. Hence, all hypotheses regarding mediation $\mathrm{H}_{7}, \mathrm{H}_{8}, \mathrm{H}_{9}, \mathrm{H}_{10}$, and $\mathrm{H}_{11}$ were accepted.

\section{Discussion and Conclusion}

According to regression analysis learning orientation of sales representatives were found to have a positive effect on sales performance $(\beta=0.132, \mathrm{p}<0.01)$. This result is in line with previous studies in sales literature (Sujan et al., 1994; Silver et al., 2006). It is important for sales representatives to have knowledge of the products they sell, competitors in the market, characteristics of the targeted customer groups and alternative offers available. Based on this outcome human resources departments of B2B firms may be recommended to measure job applicants' learning orientation levels before making recruitment decisions for sales representative positions. When measuring job applicants' learning orientation interviewing techniques may also be used in addition to standard tests.

One other finding of the study was that the intrinsic motivation of sales representatives influenced their sales performance positively $(\beta=0.149, \mathrm{p}<0.01)$. This result is in line with the findings of Weitz et al. (1986), Spiro and Weitz (1990), Oliver and Anderson (1994), and Bodla and Naeem (2014). Based on this finding managers in B2B businesses are recommended to establish a working climate which enables their sales representatives to develop beliefs that their jobs and work outcomes are important. It would be hard to develop a successful career for a sales representative unless s/he enjoys her/his job and is satisfied with her/his job. Those individuals who choose sales careers just because they view sales positions as opportunities to have access to attractive perks or benefits such as company car and various incentives may probably end up getting disappointed and may eventually leave 
their jobs. As sales profession requires a high level effort in terms of travelling, struggling with weather conditions, meeting new people and new challenges every day, intrinsic motivation is highly needed. For this reason an overconcentration on candidates' education, foreign language skills at the expense of their intrinsic motivation during recruitment may lead to hiring of inappropriate candidates for the job.

As expected hard-working was found to be positively related to sales performance ( $\beta=0.094$, $\mathrm{p}<0.10$ ). This outcome is similar to the findings of previous studies (Brown \& Peterson, 1994; Vande Walle et al., 1999; Silver et al., 2006; Chakrabarti et al., 2014b). Additionally, although results of the analyses indicate that customer orientation has a high level of correlation with sales performance ( $\mathrm{r}=0.475, \mathrm{p}<0.01$ ), it tends to lose its significance in terms of its direct effects $(\beta=-0.014, \mathrm{p}>0.10)$ during the presence of other factors. While many previous studies found positive relations between customer orientation and sales performance (Franke \& Park, 2006; Wachner et al., 2009; Schwepker \& Good, 2012) some others (Johnson et al., 2009; Homburg, 2011) did not. Goad and Jaramillo (2014) stated that the influence of customer orientation depends on the type of the customer. The results of this study also suggests that marketing orientation is crucial for sales representatives, but it its main importance lies in the long term retention of customers.

Findings regarding technical expertise variable showed similarities with customer orientation of sales representatives. Technical expertise of sales representatives was found to have insignificant effect $(\beta=-0.045, \mathrm{p}>0.10)$ in regression analysis. Sales literature in general argues that technical expertise has a positive relationship with sales performance (Plank \& Reid, 2010; Verbeke et al., 2010; Sangtani \& Murshed, 2013). In opposite vein, Ahmad et al. (2010:181-211) expressed that sales representatives who had higher technical expertise could face problems in terms of communicating benefits and value to the customer. Based on the findings of this study and the literature it may be suggested that technical expertise should be supported with other sales characteristics.

Finally, the study found that social intelligence of sales representatives influenced the sales performances of sales representatives directly. As suggested, significant relationships were found between social processing $(\beta=0.113, p<0.05)$, social skills $(\beta=0.187, p<0.01)$ and social awareness $(\beta=0.256, p<0.01)$ and sales performance. Additionally, the influence of social intelligence appears to be highly dominant that it mediates the relation between critical success factors in selling and sales performance.

Above results support the view that social intelligence is very important for sales representatives in $\mathrm{B} 2 \mathrm{~B}$ businesses. $\mathrm{B} 2 \mathrm{~B}$ firms should try to ensure the recruitment and maintenance sales staff who have high social intelligence. It is important to invest time and money on developing social intelligence of staff to ensure a constant pool of staff with social intelligence. Through continuous assessments, gaps and training needs may be regularly identified and appropriate training sessions could be developed.

Sales representatives in industrial markets are expected to act like consultants for their 
customers. For this to happen, first they need to listen to their customers actively. Sales representatives are expected to ask questions and inquire their customers' needs, wants and problems. Sales representatives are recommended not only focus on verbal explanations and cues. They are recommended to develop skills in understanding non-verbal cues which may communicate significant messages. Sales representatives are also required to handle customer objections skillfully. Rather than a using continuous defensive approach or making excuses, sales representatives are recommended to have the type of attitude which is instrumental in solving their customers' problems and adding value. A defensive approach can frustrate customers.

\section{References}

Agnihotri, R., \& Krush, M. (2015). Salesperson empathy, ethical behaviors, and sales performance: The moderating role of trust in one's manager. Journal of Personal Selling \& Sales Management, 35(2), 164-174. https://doi.org/10.1080/08853134.2015.1010541

Ahearne, M., Lam, S. K., Mathieu, J. E., \& Bolander, W. (2010). Why are some salespeople better at adapting to organizational change? Journal of Marketing, 74(3), 65-79. https://doi.org/10.1509/jmkg.74.3.65

Ahmad, S. Z., Sah, B. M., \& Kitchen, P. J. (2010). The Relationship between sales skills and salesperson performance, and the impact of organizational commitment as a moderator: An empirical study in a Malaysian telecommunications company. Int. Journal of Economics and Management, 4(2), 181-211.

Albers, S., Raman, K., \& Lee, N. (2015). Trends in optimization models of sales force management. Journal of Personal Selling \& Sales Management, 35(4), 275-291. https://doi.org/10.1080/08853134.2015.1085807

Alhouti, S., Butler, T. D., Johnson, C. M., \& Davis, L. (2014). Unwanted Pursuit Behavior: Understanding Salespeople's Desire to Pursue and Desire to Avoid Customers. Journal of Marketing Theory and Practice, 22(4), 385-400. https://doi.org/10.2753/MTP1069-6679220403

Anderson, E., \& Oliver, R. L. (1987). Perspectives on behavior-based versus outcome-based salesforce control systems. Journal of Marketing, 51(October), 76-88. https://doi.org/10.2307/1251249

Babakus, E., Cravens, D. W., Grant, K., Ingram, T. N., \& LaForge, R. W. (1996). Investigating the relationships among sales, management control, sales territory design, salesperson performance, and sales organization effectiveness. International Journal of Research in Marketing, 13(4), 345-363. https://doi.org/10.1016/S0167-8116(96)00016-X

Baron, R. M., \& Kenny, D. A. (1986). The moderator-mediator variable distinction in social psychological research: conceptual, strategic, and statistical considerations. Journal of $\begin{array}{llrl}\text { Personality and } \quad \text { Social } & \text { Psychology, } & \text { 51(6), }\end{array}$ 
https://doi.org/10.1037/0022-3514.51.6.1173

Bassett-Jones, N., \& Lloyd, G. C. (2005). Does Herzberg's motivation theory have staying power? The Journal of Management Development, 24(10), 929-943. https://doi.org/10.1108/02621710510627064

Bateman, C., \& Valentine, S. (2015). The impact of salesperson customer orientation on the evaluation of a salesperson's ethical treatment, trust in the salesperson, and intentions to purchase. Journal of Personal Selling \& Sales Management, 35(2), 125-142. https://doi.org/10.1080/08853134.2015.1010538

Behrman, D. N., \& Perreault, W. D. (1982). Measuring the performance of industrial salespersons. Journal of Business Research, 10, 355-370. https://doi.org/10.1016/0148-2963(82)90039-X

Bell, S. J., B. Mengüç, and R. E. Widing (2010). Salesperson learning, organizational learning, and retail store performance. Journal of the Academy of Marketing Science, 38, 187-201. https://doi.org/10.1007/s11747-009-0149-x

Blocker, C. P., Cannon, J., Panagopoulos, N., \& Sager, J. (2012). The role of the sales force in value creation and appropriation: New directions for research. Journal of Personal Selling \& Sales Management, 32(1), 15-28. https://doi.org/10.2753/PSS0885-3134320103

Bodla, M. A., \& Naeem, B. (2014). Creativity as Mediator for Intrinsic Motivation and Sales Performance. Creativity Research Journal, 26(4), 468-473. https://doi.org/10.1080/10400419.2014.961783

Boyer, S. L., Artis, A. B., Solomon, P. J., \& Fleming, D. E. (2012). Improving sales performance with self-directed learning. The Marketing Management Journal, 22(2), 61-75.

Boyer, S. L., Artis, A. B., Fleming, D. E., \& Solomon, P. J. (2014). The Impact of Perceived Organizational Support on Self-Directed Learning in Sales Training. Journal of Marketing Channels, 21(2), 65-76. https://doi.org/10.1080/1046669X.2013.854192

Bradford, K., Brown, S., Ganesan, S., Hunter, G., Onyemah, V., Palmatier R., et al. (2010). The embedded sales force: Connecting buying and selling organizations. Marketing Letters, 21(3), 239-253. https://doi.org/10.1007/s11002-010-9106-1

Brown, S. P., \& Peterson, R. A. (1994). The effect of effort on sales performance and job-satisfaction. Journal of Marketing, 58(2), 70-80. https://doi.org/10.2307/1252270

Calantone, R. J., Cavusgil, S. T., \& Zhao, Y. (2002). Learning orientation, firm innovation capability, and firm performance. Industrial Marketing Management, 31, 515-524. https://doi.org/10.1016/S0019-8501(01)00203-6

Castleberry, S. B., \& Shepherd, C. D. (1993). Effective interpersonal listening and personal selling. Journal of Personal Selling and Sales Management, 13(1), 35-49. 
Chakrabarti, R., Barnes, B. R., Berthon, P., Pitt, L., \& Monkhouse, L. L. (2014a). Goal orientation effects on behavior and performance: evidence from international sales agents in the Middle East. The International Journal of Human Resource Management, 25(2), 317-340. https://doi.org/10.1080/09585192.2013.826915

Chakrabarty, S., Widing, R. E., \& Brown, G. (2014b). Selling behaviors and sales performance: The moderating and mediating effects of interpersonal mentalizing. Journal of

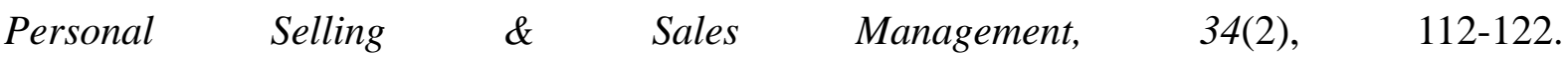
https://doi.org/10.1080/08853134.2014.890899

Chin, W. W. (1998). The partial least squares approach to structural equation modeling. In G. A. Marcoulides (Ed.), Modern Methods for Business Research (pp. 295-336). New Jersey: Lawrence Erlbaum.

Chonko, L. B., Dubinsky, A. J., Jones, E., \& Roberts, J. A. (2003). Organizational and individual learning in the sales force: an agenda for sales research. Journal of Business Research, 56, 935-946. https://doi.org/10.1016/S0148-2963(01)00330-7

Chu, W., Gerstner, E., \& Hess, J. D. (1995). Costs and benefits of hard-sell. Journal of Marketing Research, 32(1), 97-102. https://doi.org/10.2307/3152114

Churchill Jr, G. A., Ford, N. M., Hartley, S. W., Walker Jr, O. C. (1985). The determinants of salesperson performance: A meta-analysis. Journal of Marketing Research, 22(2), 103-118. https://doi.org/10.2307/3151357

Comer, L. B. (1999). Active empathetic listening and selling success: A conceptual framework. Journal of Personal Selling \& Sales Management, 19(1), 15-30.

Corsaro, D., \& Snehota, I. (2010). Searching for Relationship Value in Business Markets: Are We Missing Something? Industrial Marketing Management, 39(6), 986-995. https://doi.org/10.1016/j.indmarman.2010.06.018

Cravens, D. W., Ingram, T. N., LaForge, R. W. \& Young, C. E. (1993). Behavior-based and outcome-based salesforce control systems. Journal of Marketing, 57, 47-59. https://doi.org/10.2307/1252218

Deeter-Schmelz, D. R., \& Sojka, J. Z. (2003). Developing effective salespeople: Exploring the link between emotional intelligence and sales performance. The International Journal of Organizational Analysis, 11(3), 211-220. https://doi.org/10.1108/eb028972

Deeter-Schmelz, D. R., \& Sojka, J. Z. (2007). Personality traits and sales performance: Explorıng differential effects of need or cognition and self-monitoring. Journal of Marketing Theory and Practice, 15(2), 145-157. https://doi.org/10.2753/MTP1069-6679150204

Dweck, C. S., \& Leggett, E. L. (1988). A social-cognitive approach to motivation and personality. Psychological Review, 256-273. https://doi.org/10.1037/0033-295X.95.2.256 
Evans, K. R., McFarland, R. G., Dietz, B., \& Jaramillo, F. (2012). Advancing sales performance research: A focus on five underresearched topic areas. Journal of Personal Selling and Sales Management, 32(1), 89-106. https://doi.org/10.2753/PSS0885-3134320108

Flaherty, K. E., \& Pappas, J. M. (2004). Job selection among salespeople: A bounded rationality perspective. Industrial Marketing Management, 33(4), 325-332. https://doi.org/10.1016/S0019-8501(03)00082-8

Fornell, C., \& Larcker, D. F. (1981). Evaluating structural equation models with unobservable variables and measurement error. Journal of Marketing Research, 18(1), 39. https://doi.org/10.2307/3151312

Franke, G. R., \& Park, J. E. (2006). Salesperson adaptive selling behavior and customer orientation: A meta-analysis. Journal of Marketing Research, 43(4), 693-702. https://doi.org/10.1509/jmkr.43.4.693

Giacobbe, R. W., Jackson Jr., D. W., Crosby, L. A., \& Bridges, C. M. (2006). A contingency approach to adaptive selling behavior and sales performance: Selling situations and salesperson characteristics. Journal of Personal Selling \& Sales Management, 26(2), 115-142. https://doi.org/10.2753/PSS0885-3134260202

Goad, E. A., \& Jaramillo, F. (2014). The good, the bad and the effective: a meta-analytic examination of selling orientation and customer orientation on sales performance. Journal of Personal Selling \& Sales Management, 34 (May), 1-17. https://doi.org/10.1080/08853134.2014.899471

Goleman, D. (2006). Social intelligence: The new science of human relationships. New York: Bantam Books.

Grönroos, C. (2010). A service perspective on business relationships: The value creation, interaction and marketing interface. Industrial Marketing Management, 40(3), 240-247

Haas, A., Snehota, I., \& Corsaro, D. (2012). Creating value in business relationships: The role of sales. Industrial Marketing Management, 41(1), 94-105. https://doi.org/10.1016/j.indmarman.2011.11.004

Hair, J. F., Hult, G. T. M., Ringle, C. M., \& Sarstedt, M. (2014). A Primer on Partial Least Squares Structural Equation Modeling. Thousand Oaks: Sage.

Hair, J. F., Ringle, C. M., \& Sarstedt, M. (2011). PLS-SEM: Indeed a silver bullet. The Journal of Marketing Theory and Practice, 19(2), 139-152. https://doi.org/10.2753/MTP1069-6679190202

Hartmann, F., \& Slapničar, S. (2012). Pay fairness and intrinsic motivation: the role of pay transparency. The International Journal of Human Resource Management, 23(20), 4283-4300. https://doi.org/10.1080/09585192.2012.664962

Hayes, A. F. (2012). PROCESS: A versatile computational tool for observed variable 
mediation, moderation, and conditional process modeling. Retreived from http://www.afhayes.com/public/process2012.pdf on 12th July 2016.

Hohenschwert, L., \& Geiger, S. (2015). Interpersonal influence strategies in complex B2B sales and the socio-cognitive construction of relationship value. Industrial Marketing Management, 49, 139-150. https://doi.org/10.1016/j.indmarman.2015.05.027

Homburg, C., Wieseke, J., \& Bornemann, T. (2009). Implementing the Marketing Concept at the Employee-Customer Interface: The Role of Customer Need Knowledge. Journal of Marketing, 73(4), 64-81. https://doi.org/10.1509/jmkg.73.4.64

Hunter, G. K., \& Perreault Jr., W. D. (2007). Making sales technology effective. American Marketing Association, 71(1), 16-34. https://doi.org/10.1509/jmkg.71.1.16

Johnson, M. S., Sivadas, E., \& Kashyap, V. (2009). Response bias in the measurement of salesperson orientations: The role of impression management. Industrial Marketing Management, 38(8), 1014-1024. https://doi.org/10.1016/j.indmarman.2009.03.010

Joshi, A. W., \& Randall, S. (2001). The indirect effects of organizational controls on salesperson performance and customer orientation, Journal of Business Research, 54, 1-9.

Ko, D., \& Dennis, A. R. (2013). Sales force automation and sales performance: Do experience and expertise matter? Journal of Personal Selling \& Sales Management, 24(4) 311-322. https://doi.org/10.1016/S0148-2963(99)00119-8

Koc, E. (2003) The Role and Potential of Travel Agency Staff as a Marketing Communications Tool, Tourism Analysis-An Interdisciplinary Journal, 8(1), 105-111. https://doi.org/10.3727/108354203108750139

Koc, E. (2009) A Review of Country Competitiveness, Tourism Industry Performance and Research Performance Relationships, Competitiveness Review, 19(2), 119-133. https://doi.org/10.1108/10595420910942298

Kohli, A. K., Shervani, T. A., \& Challagalla, G. N. (1998). Learning and performance orientation of salespeople: The role of supervisors. Journal of Marketing Research, 35(May), 263-274. https://doi.org/10.2307/3151853

Krishnan, B. C., Netemeyer, R. G., Boles, J. S. (2002). Self-efficacy, competitiveness, and effort as antecedents of salesperson performance. Journal of Personal Selling \& Sales Management, 22(4), 285-295.

Latham, G. P., \& Ernst, C. T. (2006). Keys to motivating tomorrow's workforce. Human Resource Management Review, 16(2), 181-198. https://doi.org/10.1016/j.hrmr.2006.03.014

Leigh, T. W., DeCarlo, T. E., Allbright, D., \& Lollar, J. (2014). Salesperson knowledge distinctions and sales performance. Journal of Personal Selling \& Sales Management, 34(2), 123-140. https://doi.org/10.1080/08853134.2014.890902 
McFarland, R. G. (2003). Crisis of conscience: The use of coercive sales tactics and resultant felt stress in the salesperson. The Journal of Personal Selling and Sales Management, 23(4), 311-325.

Miao, C. F., \& Evans, K. R. (2012). Effects of formal sales control systems: A combinatory perspective. International Journal of Research in Marketing, 29(2), 181-191. https://doi.org/10.1016/j.ijresmar.2011.09.002

Miao, C. F., \& Evans, K. R. (2014). Motivating industrial salesforce with sales control systems: An interactive perspective. Journal of Business Research, 67(6), 1233-1242. https://doi.org/10.1016/j.jbusres.2013.04.007

Oliver, R. L., \& Anderson, E. (1994). An empirical test of the consequences of behaviour-and outcome-based sales control systems. Journal of Marketing, 58(4), 53-67. https://doi.org/10.2307/1251916

Paparoidamis, N. G., \& Guenzi, P. (2009). An empirical investigation into the impact of relationship selling and LMX on salespeople's behaviour and sales effectiveness. European Journal of Marketing, 43(7/8), 1053-1075. https://doi.org/10.1108/03090560910961515

Park, J. E., J. Kim, A. J. Dubinsky, and H. Lee (2007). How does sales force automation influence relationship quality and performance? The mediating roles of learning and selling behaviors. Industrial Marketing Management, 39, 1128-1138 https://doi.org/10.1016/j.indmarman.2009.11.003

Pettijohn, C. E., Pettijohn, L. S., \& Taylor, A. J. (2007). Does salesperson perception of the importance of sales skills improve sales performance, customer orientation, job satisfaction, and organizational commitment, and reduce turnover? Journal of Personal Selling and Sales Management, 27(1), 75-88. https://doi.org/10.2753/PSS0885-3134270105

Piercy, N. F., Cravens, D. W., \& Lane, N. (2007). Enhancing salespeople's effectiveness. Marketing Management, September/October, 18-25.

Plank, R. E., \& Reid, D. A. (2010). The interrelationships of empathy, trust and conflict and their impact on sales performance: An exploratory study. The Marketing Management Journal, 20(2), 119-139.

Rentz, J. O., Shepherd, C. D., Tashchian, A., Dabholkar, P. A., \& Ladd, R. T. (2002). A measure of selling skill: Scale development and validation. Journal of Personal Selling \& Sales Management, 22(1), 13-21.

Ribeiro, A. H. P., da Veiga, R. T., \& Robbe, R. S. (2015). Value Generation and Impact On Commitment In Business-to-Business Relationships. In Marketing Dynamism \& Sustainability: Things Change, Things Stay the Same... (pp. 776-785). Springer International Publishing.

Riggio, R. E., \& Lee, J. (2007). Emotional and interpersonal competencies and leader 
development. Human Resource Management Review, 17(4), 418-426. https://doi.org/10.1016/j.hrmr.2007.08.008

Ringle, C. M., Wende, S., \& Becker, J.-M. (2015). SmartPLS 3. Bönningstedt: SmartPLS. Retrieved from http://www.smartpls.com

Rodriguez, M., \& Honeycutt, E. D. (2011). Customer Relationship Management (CRM)'s Impact on B-to-B Sales Professionals' Collaboration and Sales Performance. Journal of Business-to-Business Marketing, 18(4), 335-356. https://doi.org/10.1080/1051712X.2011.574252

Rozell, E. J., Pettijohn, C. E., \& Parker, R. S. (2006). Emotional intelligence and dispositional affectivity as predictors of performance in salespeople. Journal of Marketing Theory and Practice, 14(2), 113-124. https://doi.org/10.2753/MTP1069-6679140202

Sabnis, G., Chatterjee, S. C., Grewal, R., \& Lilien, G. L. (2013). The Sales Lead Black Hole: On Sales Reps' Follow-Up of Marketing Leads. Journal of Marketing, 77(1), 52-67. https://doi.org/10.1509/jm.10.0047

Sangtani, V., \& Murshed, F. (2013). Product knowledge, optimism, and salesperson performance. Annals of the Society for Marketing Advances, 2, 229-233.

Saxe, R., \& Weitz, B. A. (1982). The SOCO scale: A measure of the customer orientation of salespeople. Journal of Marketing Research, 19(August),343-351. https://doi.org/10.2307/3151568

Schwepker, C. H., \& Good, D. J. (2012). Sales quotas: Unintended consequences on trust in organization, customer-oriented selling, and sales performance. The Journal of Marketing Theory and Practice, 20(4), 437-452. https://doi.org/10.2753/MTP1069-6679200406

Schwepker Jr., C. H. (2003). Customer-oriented selling: A review, extension, and directions for future research. Journal of Personal Selling \& Sales Management, 23(2), 151-171.

Silver, L. S., Dwyer, S., \& Alford, B. (2006). Learning and performance goal orientation of salespeople revisited: The role of performance-approach and performance-avoidance orientations. Journal of Personal Selling and Sales Management, 26(1), 27-38. https://doi.org/10.2753/PSS0885-3134260103

Silvera, D. H., Martinussen, M., \& Dahl, T. I. (2001). The Tromsø Social Intelligence Scale, a self-report measure of social intelligence. Scandinavian Journal of Psychology, 42(4), 313-319. https://doi.org/10.1111/1467-9450.00242

Sinkula, J., Baker, W., \& Noordewier, T. (1997). A framework for market-based organizational learning: Linking values, knowledge, and behavior. Journal of the Academy of Marketing Science, 25(4), 305-318. https://doi.org/10.1177/0092070397254003

Slater, S. F., \& Narver, J. C. (1995). Market orientation and the learning organization. Journal of Marketing, 59(July), 63-74. https://doi.org/10.2307/1252120 
Spiro, R. L., \& Weitz, B. A. (1990). Conceptualization, adaptive selling: measurement, nomological validity. Journal of Marketing Research, 27(1), 61-69. https://doi.org/10.2307/3172551

Strong, C. A., \& Harris, L. C. (2004). The drivers of customer orientation: An exploration of relational, human resource and procedural tactics. Journal of Strategic Marketing, 12(3), 183-204. https://doi.org/10.1080/0965254042000262904

Sujan, H. (1986). Smarter versus harder: An exploratory attributional analysis of salespeople's motivation. Journal of Marketing Research, 23(1), 41-49. https://doi.org/10.2307/3151775

Sujan, H., Weitz, B. A., \& Kumar, N. (1994). Learning orientation, working smart, and effective selling. Journal of Marketing, 58(July), 39-52. https://doi.org/10.2307/1252309

Sujan, H., Weitz, B., \& Sujan, M. (1988). Increasing sales productivity by getting salespeople to work smarter. Journal of Personal Selling \& Sales Management, 8(2), 9-19.

Terho, H., Eggert, A., Haas, A., \& Ulaga, W. (2015). How sales strategy translates into performance: The role of salesperson customer orientation and value-based selling. Industrial Marketing Management, 45, 12-21. https://doi.org/10.1016/j.indmarman.2015.02.017

VandeWalle, D., Brown, S. P., Cron, W. L., \& Slocum, J. W. (1999). The influence of goal orientation and self-regulation tactics on sales performance: A longitudinal field test. Journal of Applied Psychology, 84(2), 249-259. https://doi.org/10.1037/0021-9010.84.2.249

Verbeke, W., Dietz, B., \& Verwaal, E. (2010). Drivers of sales performance: A contemporary meta-analysis. Journal of the Academy of Marketing Science, 39(3), 407-428. https://doi.org/10.1007/s11747-010-0211-8

Vieira, A. L., Winklhofer, H., \& Ennew, C. (2014). The Effects of Relationship Marketing on Share of Business: A Synthesis and Comparison of Models. Journal of Business-to-Business Marketing, 21(2), 85-110. https://doi.org/10.1080/1051712X.2014.903455

Wachner, T., Plouffe, C. R., \& Grégoire, Y. (2009). SOCO’s impact on individual sales performance: The integration of selling skills as a missing link. Industrial Marketing Management, 38(1), 32-44. https://doi.org/10.1016/j.indmarman.2007.11.003

Walker, O. C., Churchill, G. A, \& Ford, N. M. (1977). Motivation and performance in industrial selling. Journal of Marketing Research, 14(2), 156-168. https://doi.org/10.2307/3150465

Wang, G., \& Netemeyer, R. G. (2002). The effects of job autonomy, customer demandingness, and trait competitiveness on salesperson learning, self-efficacy, and performance. Journal of the Academy of Marketing Science, 30(3), 217-228. https://doi.org/10.1177/0092070302303003

Wawra, D. (2009). Social Intelligence, the key to intercultural communication. European 
Journal of English Studies, 13(2), 163-177. https://doi.org/10.1080/13825570902907193

Wei, Y. S., Samiee, S., \& Lee, R. P. (2014). The influence of organic organizational cultures, market responsiveness, and product strategy on firm performance in an emerging market. Journal of the Academy of Marketing Science, 42(1), 49-70. https://doi.org/10.1007/s11747-013-0337-6

Weitz, B. A., Sujan, H., \& Sujan, M. (1986). Knowledge, motivation, and adaptive behavior: A framework for improving selling effectiveness. Journal of Marketing, 50(October), 174-191. https://doi.org/10.2307/1251294

Yu, T., Patterson, P., \& de Ruyter, K. (2015). Converting service encounters into cross-selling opportunities: does faith in supervisor ability help or hinder service-sales ambidexterity? European Journal of Marketing, 49(3/4), 491-511. https://doi.org/10.1108/EJM-10-2013-0549

Zampetakis, L. A. (2014). Sales force management practices in organizations with a supportive climate towards creativity. Journal of Strategic Marketing, 22(1), 59-72. https://doi.org/10.1108/EJM-10-2013-0549

Zhou, W., Hu, H., \& Shi, X. (2015). Does organizational learning lead to higher firm performance? An investigation of Chinese listing companies. The Learning Organization, 22(5), 271-288. https://doi.org/10.1108/TLO-10-2012-0061

\section{Appendix}

Appendix 1. Confirmatory Factor Analysis Factor Loadings

\begin{tabular}{|l|l|l|}
\hline & Scale & $\begin{array}{l}\text { Factor } \\
\text { Loadings }\end{array}$ \\
\hline & Sales Performance & \\
\hline 1 & Contributing to your company's acquiring a good market share. & 0.692 \\
\hline 2 & Selling high profit margin products. & 0.739 \\
\hline 3 & Generating a high level of dollar sales. & 0.789 \\
\hline 4 & Quickly generating sales of new company products. & 0.799 \\
\hline 5 & Identifying major accounts in your territory and selling them. & 0.762 \\
\hline 6 & Exceeding sales targets. & 0.614 \\
\hline 7 & Assisting your sales supervisor to meet his or her goals. & 0.773 \\
\hline & Learning Orientation & 0.797 \\
\hline 1 & $\begin{array}{l}\text { An important part of being a good salesperson is continually improving } \\
\text { your sales skills }\end{array}$ & 0.809 \\
\hline 2 & It is important for me to learn from each selling experience I have & 0.721 \\
\hline 5 & $\begin{array}{l}\text { It is worth spending a great deal of time learning new approaches for } \\
\text { dealing with customers }\end{array}$ & 0.790 \\
\hline 6 & Learning how to be a better salesperson is of fundamental importance to me & 0.710 \\
\hline 7 & I put in a great deal of effort sometimes to learn something new & 0.794 \\
\hline & Customer Orientation & 0.704 \\
\hline 2 & $\begin{array}{l}\text { I always present the customer with a realistic picture of what my firm's } \\
\text { products can do. }\end{array}$ & $\begin{array}{l}\text { I spend much of my time listening to the customer talk about his/her firm's } \\
\text { needs. }\end{array}$ \\
\hline
\end{tabular}


4 I wait until I fully understand the customer's needs before making my sales presentation.

0.822

5 I am always candid in discussions with my customers.

Intrinsic Motivation

2 I sell because of the feeling of performing a useful service

3 I obtain a sense of accomplishment from my work

4 I feel a sense of personal growth and development in my work

Hard-working

1 I work long hours to meet my sales objectives.

0.666

2 I do not give up easily when I encounter a customer who is difficult to sell.

3 I work untiringly at selling a customer until I get an order. Technical Expertise

2 I have knowledge of my company's procedures.

0.814

0.905

0.825

0.725

\begin{tabular}{l|l|l}
3 & I have knowledge of our competitors' products, services, and sales policies. & 0.817
\end{tabular}

4 I have knowledge of our product line, including product features and

benefits.

5 I have knowledge of my customers' operations

0.702

6 I have imagination in supplying products and services that meet my customers' needs.

0.709

0.654

Confirmatory Factor Analysis Factor Loadings

\begin{tabular}{|c|c|c|c|c|}
\hline & Social Intelligence Scale & \multicolumn{3}{|c|}{ Factor Loadings } \\
\hline & & F1 & F2 & F3 \\
\hline 1 & I can predict other peoples' behavior. & 0.707 & 0.421 & 0.380 \\
\hline 2 & I know how my actions will make others feel. & 0.783 & 0.478 & 0.470 \\
\hline 6 & I understand other peoples’ feelings. & 0.776 & 0.553 & 0.430 \\
\hline 9 & I understand others' wishes. & 0.776 & 0.553 & 0.511 \\
\hline 14 & $\begin{array}{l}\text { I can often understand what others are trying to accomplish without } \\
\text { the need for them to say anything. }\end{array}$ & 0.716 & 0.342 & 0.442 \\
\hline 17 & I can predict how others will react to my behavior. & 0.753 & 0.411 & 0.426 \\
\hline 19 & $\begin{array}{l}\text { I can often understand what others really mean through their } \\
\text { expression, body language, etc. }\end{array}$ & 0.794 & 0.568 & 0.475 \\
\hline 4 & I often feel uncertain around new people who I don't know. & 0.524 & 0.799 & 0.410 \\
\hline 7 & I fit in easily in social situations. & 0.514 & 0.851 & 0.481 \\
\hline 10 & $\begin{array}{l}\text { I am good at entering new situations and meeting people for the first } \\
\text { time. }\end{array}$ & 0.497 & 0.848 & 0.484 \\
\hline 12 & I have a hard time getting along with other people. & 0.465 & 0.751 & 0.405 \\
\hline 15 & It takes a long time for me to get to know others well. & 0.532 & 0.807 & 0.418 \\
\hline 18 & I am good at getting on good terms with new people. & 0.553 & 0.800 & 0.533 \\
\hline 20 & I frequently have problems finding good conversation topics. & 0.470 & 0.756 & 0.465 \\
\hline 3 & I often feel that it is difficult to understand others' choices. & 0.532 & 0.467 & 0.850 \\
\hline 5 & People often surprise me with the things they do. & 0.470 & 0.538 & 0.805 \\
\hline 8 & $\begin{array}{l}\text { Other people become angry with me without me being able to explain } \\
\text { why. }\end{array}$ & 0.344 & 0.421 & 0.691 \\
\hline 11 & $\begin{array}{l}\text { It seems as though people are often angry or irritated with me when I } \\
\text { say what I think. }\end{array}$ & 0.424 & 0.375 & 0.791 \\
\hline 13 & I find people unpredictable. & 0.497 & 0.457 & 0.838 \\
\hline 16 & I have often hurt others without realizing it. & 0.483 & 0.450 & 0.745 \\
\hline 21 & I am often surprised by others' reactions to what I do. & 0.533 & 0.469 & 0.850 \\
\hline & chion & & & \\
\hline
\end{tabular}




\section{Copyright Disclaimer}

Copyright for this article is retained by the author(s), with first publication rights granted to the journal.

This is an open-access article distributed under the terms and conditions of the Creative Commons Attribution license (http://creativecommons.org/licenses/by/3.0/). 